\title{
Preliminary Study on Ultrasonic Ageing Zhenjiang Vinegar Mechanism Based on Maillard Simulation System
}

\author{
Xiaoke Ma, ${ }^{1}$ Tingting Li $\left(D,{ }^{2}\right.$ Yuanqing He, ${ }^{3,4}$ Min Chen, ${ }^{3}$ Jie Zhou, ${ }^{4}$ Lijing Yin, ${ }^{4}$ \\ and Haile $\mathrm{Ma}^{3}{ }^{3}$ \\ ${ }^{1}$ Institute of Life Sciences, Jiangsu University, 301 Xuefu Road, Zhenjiang 212013, China \\ ${ }^{2}$ SinoUnison Technology Co. Ltd., Shandong Branch, No. 8 Building, No. 168 Zhuzhou Road Laoshan, Qingdao, \\ Shandong Province, China \\ ${ }^{3}$ School of Food and Biological Engineering, Jiangsu University, 301 Xuefu Road, Zhenjiang 212013, China \\ ${ }^{4}$ The Laboratory Animal Research Center, Jiangsu University, Zhenjiang 212013, China \\ Correspondence should be addressed to Tingting Li; ytlitingting@163.com and Haile Ma; mhl@ujs.edu.cn
}

Received 14 March 2020; Revised 13 May 2020; Accepted 16 September 2020; Published 5 October 2020

Academic Editor: Quancai Sun

Copyright (C) 2020 Xiaoke Ma et al. This is an open access article distributed under the Creative Commons Attribution License, which permits unrestricted use, distribution, and reproduction in any medium, provided the original work is properly cited.

\begin{abstract}
In this study, ultrasonic technology was used to treat Zhenjiang vinegar, and the effects on the physicochemical characteristics of Zhenjiang vinegar were investigated. The influences of ultrasound time and power on the number of induced hydroxyl radicals and superoxide radicals were also investigated. Besides, the novel simulation system of the Maillard reaction was built to research the effects of different ultrasonic times and power treatment on Zhenjiang vinegar. The results show that, under the conditions of ultrasonic treatment, the changes of Zhenjiang vinegar physiochemical index, such as color, reducing sugar, and amino acid, are consistent with those of natural ageing. In addition, ultrasound can produce a cavitation effect and cracking water molecules to produce hydroxyl radicals and superoxide radicals, so as to achieve the ageing effect of vinegar.
\end{abstract}

\section{Introduction}

Fermented vinegar has widely been used in China and other Asian countries as a food condiment for hundreds of years $[1,2]$. Zhenjiang Vinegar, which is one of the most famous traditional fermented vinegar types in China, is world-famous for its special elegant and complex aroma [3]. It is widely accepted like wine because of the harsh taste, pungent smell, and some possible harmful side-effects; fresh vinegar needs a specific process known as ageing in which a specific time period was used to produce high-quality vinegar [4]. Traditionally, ageing vinegar in barrel ageing systems is a common practice used for improving vinegar quality and stability. However, considering the disadvantages of natural ageing such as high production cost and long time period, it is critical and essential to study innovative ageing technology.

Recently, scientists have put great attempts into promoting brand new ageing techniques and enhancing present techniques. Present studies showed that there are diverse physical methods displaying considerable potential for accelerating the ageing process, including ultrasonic wave, electric fields, ultra-high pressure, and gamma irradiation [5-10]. It was found that the ultrasound $(16-60 \mathrm{kHz})$ was adept to expedite oxidation, condensation, and polymerization of ethanol, aldehydes, esters, and olefins in wines [11]. Zheng et al. [10] found an optimum treatment for speeding up fresh red wines ageing, which enhanced wines with excellent taste and flavor. Reference [12] found that the high-pressure intervention in the scope from $80 \mathrm{MPa}$ to $120 \mathrm{MPa}$ not beyond 2 hours was shown to considerably improve the wine taste. Better apprehension of each divisor that impacts the quality of vinegar ageing process combined the economic and operational reasons; ultrasonic is a potential physical method to accelerate the vinegar ageing process [13].

Ultrasound, especially with high power and low frequency, has the advantage of pollution-free and being speedy. Over the last decades, abundant works have 
concentrated on the utilization of ultrasound for food processing, like extraction, freezing, oxidization, sterilisation, and desiccation $[14,15]$. Evidence from different research [16-18] showed that the influence of ultrasound on vinegar ageing processing was possibly associated with the acoustic cavitation $[19,20]$, which includes the generation, expansion, and breakdown of microbubbles. The violent breakdown of bubbles can generate exceedingly steep heat and pressure. Many scientists have studied that during ageing processing, the vinegar undergoes many complicated changes, including Maillard reaction, oxidation reaction, and esterification reaction. Pripis-Nicolau et al. [21] revealed that, under the appropriate wine conditions, the production of odoriferous compositions or strong-smelling by-products would enhance Strecker and Maillard reactions. Casale et al. Reference [22] found that the reaction of oxidization occurs and oxygen boosts a series of chemical and enzymatic reactions that change the vinegar; thus they found an affinity between spectral variables and store time-changes in vinegar. Maillard reaction is defined as the interaction of carbonyl compounds (e.g., reducing sugars) with free amino groups (usually amino acid) leading to flavor compounds and melanoidins [23]. Maillard reaction is of great importance in food quality, especially in heat-process foods, affecting not only the flavor but also the color and nutritional values. Previous work [9] in our laboratory showed that vinegar treated with ultrasound was concluded to be tantamount to 2-3 years aged traditionally Zhenjiang vinegar under the optimum experimental conditions (ultrasonic power $50 \mathrm{~W} / 100 \mathrm{~mL}$, time $75 \mathrm{~min}$, and ethanol addition $0.75 \%(\mathrm{~V} / \mathrm{V}))$. Nevertheless, little work has been done with Zhenjiang vinegar, especially the mechanism of the chemical reactions during the ageing process.

In view of the wide variety of substance in vinegar, Maillard reactions develop complex intermediates and final reaction products, in order to clarify each substance in the ultrasound induced by the specific changes and the mechanisms is very difficult or even almost impossible. Therefore, the complex vinegar system was replaced for the simulation system $[21,24,25]$, to study the mechanism of ultrasound to accelerate the ageing process. Thus, the aim of this study was to investigate the relevant physicochemical indicators and the ageing mechanism based on the Zhenjiang vinegar simulation system. This study is of practicable interest since it provides beneficial information for vinegar industry in order to optimize the process of vinegar.

\section{Materials and Methods}

2.1. Chemicals. Chemicals used in this study were furfural, n-Octane, fructose, glycine, methylene blue, vanillin, anhydrous ethanol, and aniline which were purchased from Sinopharm Group Chemical Co., Ltd. In this study, all chemicals and solvents used were of analytical grade.

2.2. Samples. The simulated Maillard reaction system was constructed to be $100 \mathrm{~mL}$ of $0.1 \mathrm{~mol} / \mathrm{L}$ fructose and glycine aqueous solution. All the vinegar samples of different storing times used in this study were collected from Jiangsu Hengshun Vinegar Co., Ltd. Samples of vinegar were divided into 2 kinds of different treatments. One is the naturally aged vinegar samples, which were aged traditionally for different periods such as 12, 18, 32, and 44 months and kept in glasses. The samples of fresh and aged vinegar were stored at $4^{\circ} \mathrm{C}$ in a refrigerator. Samples with ultrasonic were treated according to the method of Wang et al. [9].

2.3. Color Evaluation. Color is one significant sensory characteristic of vinegar, which affects the consumers' overall acceptableness. The change of color can well reflect the process of Maillard reaction. Color measurement is measured by Hunterlab Spectrophotometer. The $L^{*}, a^{*}$, and $b^{*}$ values (CIELAB parameters) were monitored by using the software CromaLab [26], which better test the color of wines and permit better differentiation [27-30]. In this study, the CIE Lab system was used to define the color of vinegar. Record the color difference value of the vinegar $L^{*}, a^{*}$, and $b^{*}$, measured three times, and take the average. Value of $L^{*}$ indicates the brightness, the value of $0-100$; the greater the value the greater the brightness; $a^{*}$ represents the values of $\mathrm{red} /$ green, positive partial red, and negative partial green; $b^{*}$ represents the yellow/blue value, positive yellow, and negative blue. The value $\Delta E^{*}$ is measured as the Euclidean distance between two points in the three-dimensional space defined by $L^{*}, a^{*}$, and $b^{*}$ and tells from color differences:

$$
\Delta E^{*}=\sqrt{\left(\Delta L^{*}\right)^{2}+\left(\Delta a^{*}\right)^{2}+\left(\Delta b^{*}\right)^{2}} .
$$

2.4. Determination of Reducing Sugar. The content of reducing sugar in the samples was analyzed as stated in the GB/T 5009.7-2016 method including different ages of traditional aged vinegar and optimum ultrasonic experimental conditions treated vinegar (ultrasonic power $50 \mathrm{~W} / 100 \mathrm{~mL}$, time $75 \mathrm{~min}$, and ethanol addition $0.75 \%(\mathrm{~V} / \mathrm{V}))$. All vinegar samples were determined in triplicate.

2.5. Determination of Free Amino Acid. Automatic amino acid analyzer was exploited to measure the content of free amino acids in the vinegar samples. The vinegar sample to be tested was diluted 20 times with $1 \%$ sulfosalicylic acid solution, centrifuged at $10000 \mathrm{rpm}$ for $15 \mathrm{~min}$, and the supernatant was clarified through $0.22 \mu \mathrm{mmicroporous}$ membrane filter for the determination of the use of the machine.

Amino acid analysis chromatographic conditions are fluorescence detector, $4.0 \times 125 \mathrm{~mm}$ C18 column; column temperature, $40^{\circ} \mathrm{C}$; flow rate, $1.0 \mathrm{~mL} / \mathrm{min}$; and detection wavelength, Ex $440 \mathrm{~nm}$, Em $570 \mathrm{~nm}$.

2.6. Determination of Hydroxyl Radicals. The hydroxyl radical evoked by the cavitation of ultrasound was assessed indirectly by using methylene blue as the radical scavenger and ultraviolet-visible spectrophotometer as the testing method. [31, 32] The methylene blue was handled with 
dissimilar ultrasonic treatments and the maximum absorption wavelength was measured by UV-visible spectrophotometer full-wavelength scanning. The hydroxyl radical produced by ultrasound was also measured by the decrease of absorbance at the maximum absorption wavelength.

2.7. Determination of Superoxide Radicals. Vanillin aniline fluorescent reagent synthesis method: $1.5 \mathrm{~g}$ vanillin was dissolved in $20 \mathrm{~mL}$ of anhydrous ethanol; $0.9 \mathrm{~g}$ of aniline was added dropwise, refluxed at $80^{\circ} \mathrm{C}$, for $5 \mathrm{~h}$; most of the solvent was distilled off; and a pale yellow solid was obtained after cooling. Recrystallize from anhydrous ethanol and vacuum dry. $2 \times 10^{-4} \mathrm{~mol} / \mathrm{L}$ vanillin aniline ethanol solution was configured.

In a $10 \mathrm{~mL}$ colorimetric tube, add $2 \mathrm{~mL}$ of vanillin aniline ethanol solution, $2 \mathrm{~mL}$ of sonicated aqueous solution, and $1.5 \mathrm{~mL}$ of Tris-HCL buffer solution and bring the volume to the mark. The fluorescence intensity was measured under the excitation wavelength of $282 \mathrm{~nm}$, and the emission wavelength range was $250-400 \mathrm{~nm}$. Excitation and emission slit widths were measured at $5 \mathrm{~nm}$ and $10 \mathrm{~nm}$, respectively.

2.8. Determination of Maillard Reaction Process. In view of a large variety of components in vinegar, it is difficult or even impossible to find out the specific changes and mechanisms of each substance under ultrasonic induction; therefore, the content of vinegar should be higher when studying the ultrasonic accelerated vinegar ageing mechanism. The representative substances that have an important influence on the quality of vinegar were studied as objects. The results of this study are also easy to compare with the relatively clear mechanism of change in the natural maturation process of these substances. The Maillard reaction is one of the most important reactions in the vinegar ageing process. Therefore, it is necessary to construct the Maillard simulation system to study the mechanism of ultrasound on its action.

Maillard reaction model system is the $100 \mathrm{~mL}$ of $0.1 \mathrm{~mol} /$ $\mathrm{L}$ fructose and glycine solution. The degree of browning and the amount of intermediate product of the Maillard reaction were measured according to the way of $\mathrm{Gu}$ et al. [33]. According to the literature, absorbance at $294 \mathrm{~nm}$ was served to examine the formation of the intermediate compounds of the Maillard reaction and the size of the UV-visible absorption at $420 \mathrm{~nm}$ in the Maillard reaction system is related to the final stages of the reactions $[34,35]$. The browning indices were evaluated by spectrophotometry, recording the absorbance at $420 \mathrm{~nm}$ and $294 \mathrm{~nm}\left(A_{420 \mathrm{~nm}}\right.$ and $\left.A_{294 \mathrm{~nm}}\right)$ of each model system against distilled water. The results were recorded on a UV software (Beijing Beifen-Ruili) spectrophotometer using a $1 \mathrm{~cm}$ path length quartz cell. All samples were done three times in parallel.

2.9. Data Analysis. All model systems were prepared in duplicate and the analysis was performed in triplicate. Excel 2010, Origin 8.0, and SPSS Statistics 17.0 were applied to complete the data analysis. The significant differences between the different samples were obtained through a oneway ANOVA analysis with a level of $P<0.05$.

\section{Results and Discussion}

3.1. Color Evaluation. As Table 1 shows, the color of the vinegar was significantly affected by the ageing time and different storage times have a significant effect on vinegar $L^{*}$, $a^{*}, b^{*}$. For the traditionally natural aged vinegar samples with the extension of storage time, the values showed a downward trend. Lightness is the attribute of a visual sensation according to which a given visual stimulus appears to be more or less light, ranging from "light" to "dark" Gómez-m Guez [26]. Table 1 shows that all the $L^{*}$ values of vinegar samples were investigated varying from 2.07 to 0.49 units; $a^{*}$ values were between 5.54 and 1.12 units. Besides, $b^{*}$ values were between 4.21 and 0.95 . According to Oszmianski $[36,37]$, these minute dissimilarities between the vinegar samples were by reason to the oxidation instead of the enzymatic treatment. However, the previous literature $[38,39]$ reported that the ultrasound can accelerate oxidation, esterification, and other reactions. The values of the ultrasonic treated vinegar $\left(L^{*}=2.41, a^{*}=6.37, b^{*}=5.25\right)$ were higher as compared to nonultrasonic treated vinegar. The value of $\Delta E^{*}$ was found between the ultrasonic treated vinegar and fresh vinegar $\left(\Delta E^{*}=0.94\right)$. These colorimetric data present that the ultrasonic treated vinegar samples exhibited mainly red and yellow tint, which is also a consumer favor. The result shows that a tester can distinguish the color of these vinegar samples. In addition, the reducing sugar in vinegar declining with the extension of ageing time, combined with the later experimental verification Maillard reaction products, increased after ultrasonic treatment, which is consistent with the results of the color change of vinegar treatment.

3.2. Effect of Ultrasound on Hydroxyl Radicals. Figures 1 and 2 show the methylene blue solution UV-visible absorption curve along with the change of ultrasonic time and power. Besides, these results showed that methylene blue solution has the maximum absorption peak at $664 \mathrm{~nm}$ and gradually decreases with the increase of ultrasonic time and power. These results show that the concentration of methylene blue solution gradually decreased to prove that the ultrasonic treatment of hydroxyl radicals generated and hydroxyl radical concentration gradually increased over ultrasonic time and power.

As shown in Figure 3, it is not hard to see that in the initial period of time, the production of hydroxyl radical increased rapidly. As the time extended to 75 min later, the rate of increase slowed down. The reason here might be that the increased time for ultrasonic treatment increases the vinegar temperature too high. The vapor pressure in bubbles increases and consequently bubbles closed to enhance the buffer effect and the cavitation role becomes weakened. Therefore, in this study, the ultrasonic time is $75 \mathrm{~min}$.

As shown in Figure 4, with the increase of ultrasonic power, the intensity of the ultrasonic wave generated in unit 
TABLE 1: Effect of color change and reducing sugar in the natural ageing process of vinegar and ultrasound treated vinegar.

\begin{tabular}{|c|c|c|c|c|c|}
\hline \multirow{2}{*}{ Age (month) } & \multicolumn{4}{|c|}{ CIE lab color change } & \multirow{2}{*}{ Reducing sugar $(\mathrm{g} / 100 \mathrm{~mL})$} \\
\hline & $L^{*}$ & $a^{*}$ & $b^{*}$ & $\Delta E^{*}$ & \\
\hline 0 & $2.07 \pm 0.38^{\mathrm{c}}$ & $5.54 \pm 0.17^{\mathrm{d}}$ & $4.21 \pm 0.23^{\mathrm{dc}}$ & 0.00 & $4.06 \pm 0.15^{\mathrm{d}}$ \\
\hline 12 & $1.24 \pm 0.24^{\mathrm{b}}$ & $5.31 \pm 0.20^{\mathrm{d}}$ & $3.23 \pm 0.10^{\mathrm{dc}}$ & 0.85 & $3.39 \pm 0.14^{\mathrm{c}}$ \\
\hline 18 & $0.89 \pm 0.02^{\mathrm{b}}$ & $4.56 \pm 0.13^{c}$ & $2.05 \pm 0.11^{\mathrm{b}}$ & 1.03 & $2.51 \pm 0.16^{\mathrm{ab}}$ \\
\hline 32 & $0.64 \pm 0.04^{\mathrm{a}}$ & $2.22 \pm 0.11^{\mathrm{b}}$ & $1.83 \pm 0.09^{\mathrm{b}}$ & 2.79 & $2.92 \pm 0.18^{\mathrm{b}}$ \\
\hline 44 & $0.49 \pm 0.02^{\mathrm{d}}$ & $1.12 \pm 0.02^{\mathrm{a}}$ & $0.95 \pm 0.02^{\mathrm{a}}$ & 1.00 & $2.24 \pm 0.14^{\mathrm{a}}$ \\
\hline Ultrasonic treated & $2.41 \pm 0.01^{\mathrm{d}}$ & $6.37 \pm 0.02^{\mathrm{d}}$ & $5.25 \pm 0.13^{\mathrm{d}}$ & 0.94 & $3.03 \pm 0.45^{\mathrm{c}}$ \\
\hline
\end{tabular}

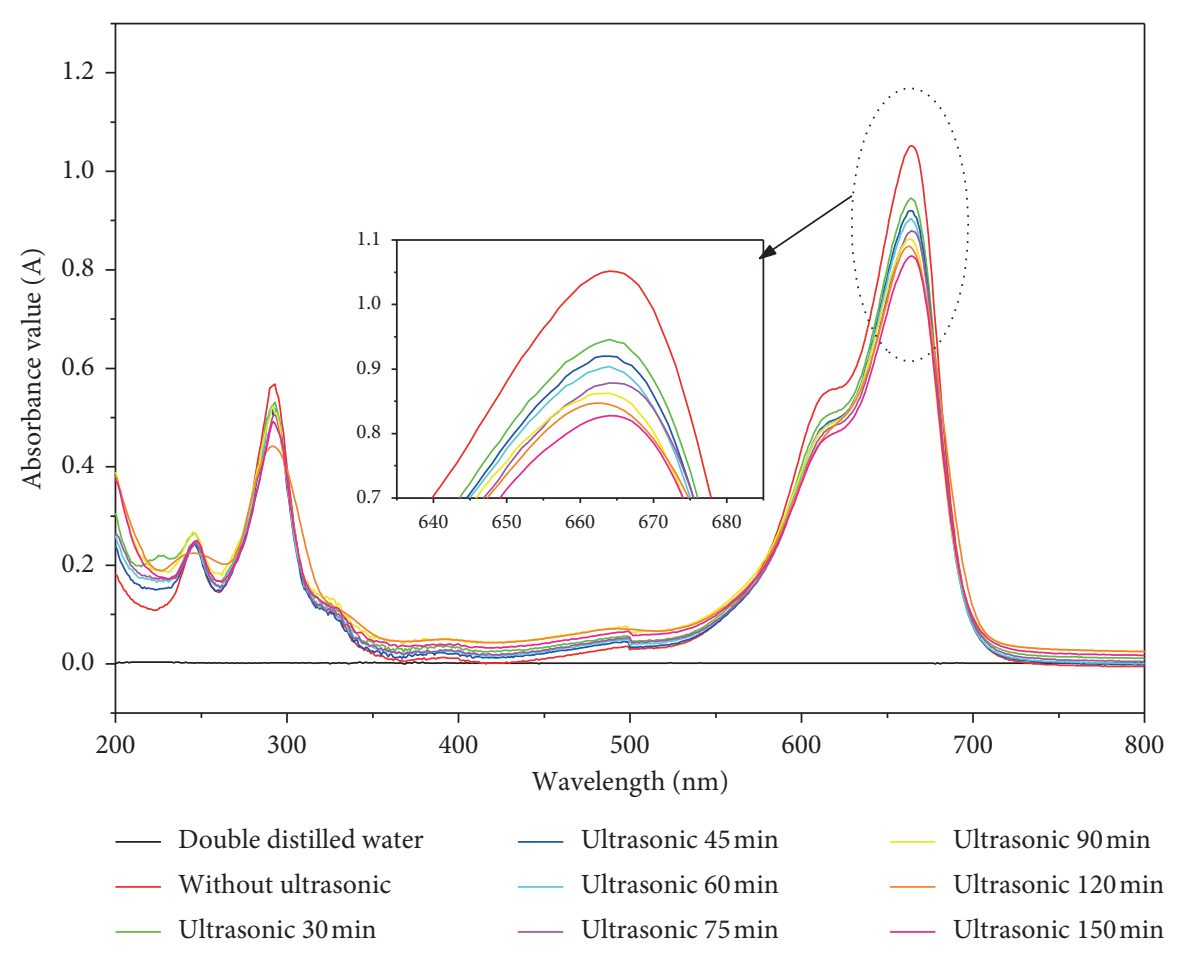

Figure 1: UV-visible spectra of methylene blue solution at different ultrasonic times.

time increases, and the cavitation effect produced is stronger [40]. Therefore, when the cavitation bubbles generated by the aqueous solution are crushed, high temperature and high-pressure environment occurs; the number of free radicals generated will be increased sufficiently.

Hydroxyl radical is the most active oxygen free radical [41]. Ultrasound-induced hydroxyl radicals can cause oxidation of sugars, amino acids, proteins, and esters in vinegar [42]. The production of free radicals can speed up the breakage and formation of various substances in vinegar, accelerate the ripening of vinegar, and shorten the ageing time [43].

3.3. Effect of Ultrasound on Superoxide Radica. From Figures 5 and 6 , it can be seen that superoxide radicals in the solution that has not been sonicated are not detected; however, a large number of free radicals are generated in the aqueous solution after sonication, which proves that the ultrasonic treatment produces superoxide radicals in the solution and has a significant effect. According to Figure 5, the number of superoxide radicals generated increases with the increase of the ultrasound time and reaches the maximum level at $40 \mathrm{~min}$ after sonication. Figure 6 shows that the number of superoxide radicals produced by ultrasound gradually decreases with the increase of ultrasonic power of $10 \mathrm{~W} / 100 \mathrm{~mL}$. The generation of superoxide radicals is due to the action of ultrasound on the oxygen molecules in the aqueous solution. The reason for the above research results is that, on the one hand, it is finally converted into hydroxyl radicals through a series of reactions which is consistent with the results of the previous studies on hydroxyl radicals and, on the other hand, superoxide anions can dismutate to produce hydrogen peroxide and oxygen [44].

Superoxide radicals in water can be considered as a base, which can accept $\mathrm{H}^{+}$from a protonated superoxide radical HOO•. In one way, the mechanism is largely to convert it into other more active oxygen ions. The base achieves the promotion of chemical reaction.

3.4. Effect of Ultrasound on Browning Degree of Maillard Reaction. Table 1 shows that after the ultrasonic treatment, the color of the vinegar becomes lighter. The content of 


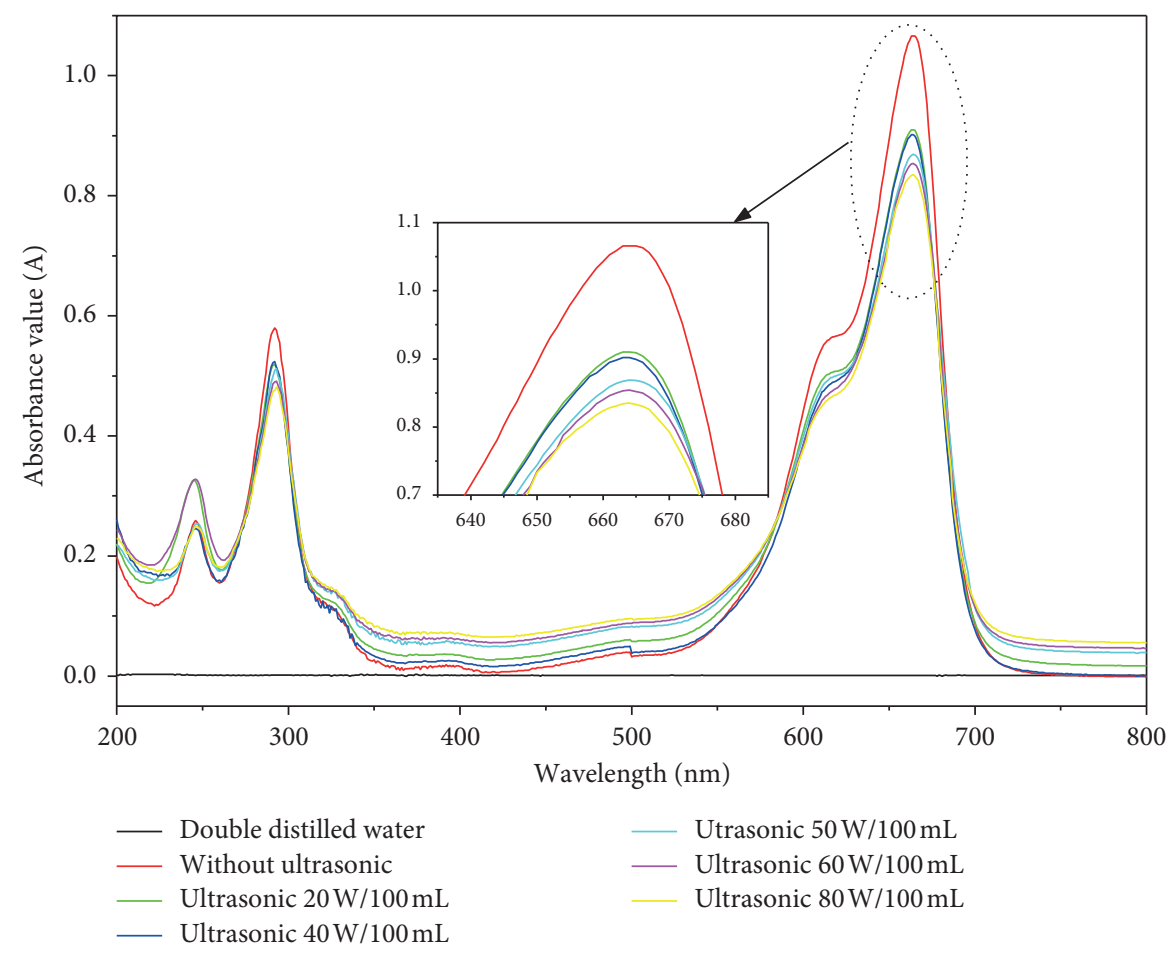

FIGURE 2: UV-visible spectra of methylene blue solution at different ultrasonic powers.

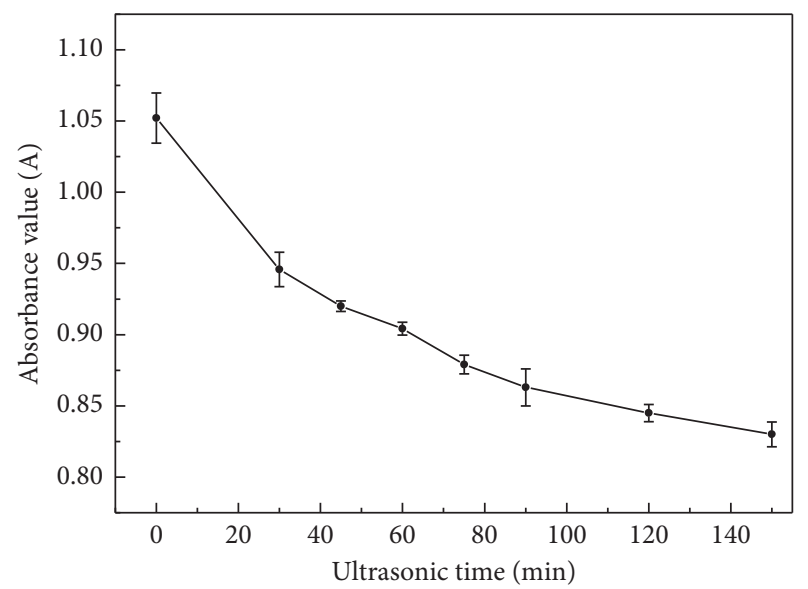

FIgURE 3: The maximum absorption intensity $(664 \mathrm{~nm})$ of the methylene blue solution in the UV-visible light varies with the ultrasonic time.

reducing sugar decreased by nearly $50 \%$ from $4.06 \mathrm{~g} / 100 \mathrm{~mL}$ to $2.24 \mathrm{~g} / 100 \mathrm{~mL}$. Besides, during the traditionally natural ageing process, the changes of vinegar free amino acids are shown in Table 2. It is revealed that as the ageing time becomes longer, the content of free amino acids decreased by $1041.35 \mathrm{mg} / 100 \mathrm{~mL}$ down to $757.87 \mathrm{mg} / 100 \mathrm{~mL}$. Moreover, after ultrasonic treatment, the content of amino acids in vinegar decreased, consistent with the trend of natural ageing. And ultrasound can produce lots of hydroxyl radicals. Combined with the above experimental results analysis, we speculate that the changes are related to the progress of the Maillard reaction. The monosaccharides in Zhenjiang vinegar is mainly glucose and fructose. And glycine in the

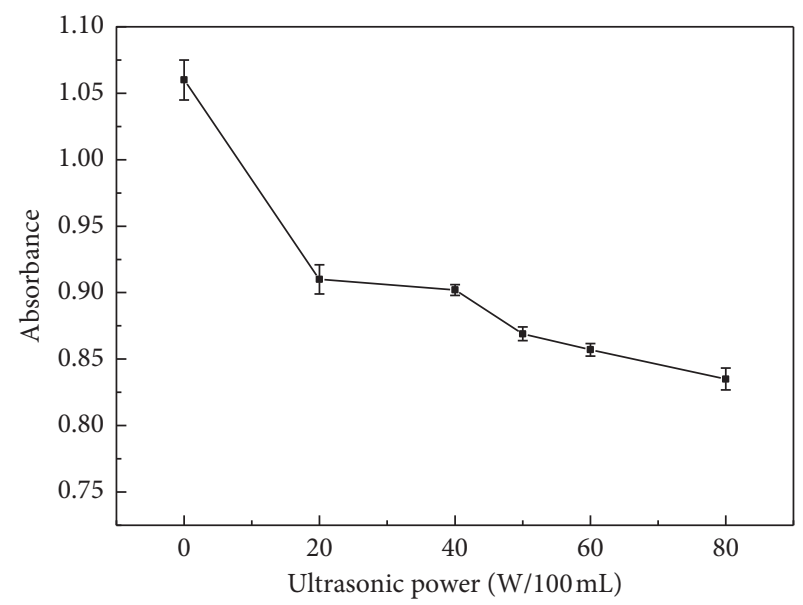

Figure 4: The maximum absorption intensity $(664 \mathrm{~nm})$ of the methylene blue solution in the UV-visible light varies with the ultrasonic power.

vinegar free amino acid mass fraction is relatively high. In particular, fructose-glycine can form a kind of Amadori compounds $[45,46]$. Therefore, in this experiment, the fructose-glycine simulation system was used to verify that ultrasound may speed up the Maillard reaction. As far as we know, the melanoidin produced during the Maillard reaction can make the color of the vinegar darker, so that the degree of browning can be directed to the extent of the Maillard reaction.

As can be seen from Figure 7, the degree of browning of the fructose-glycine simulation system gradually increased with the ultrasonic time rise. And the results showed that 


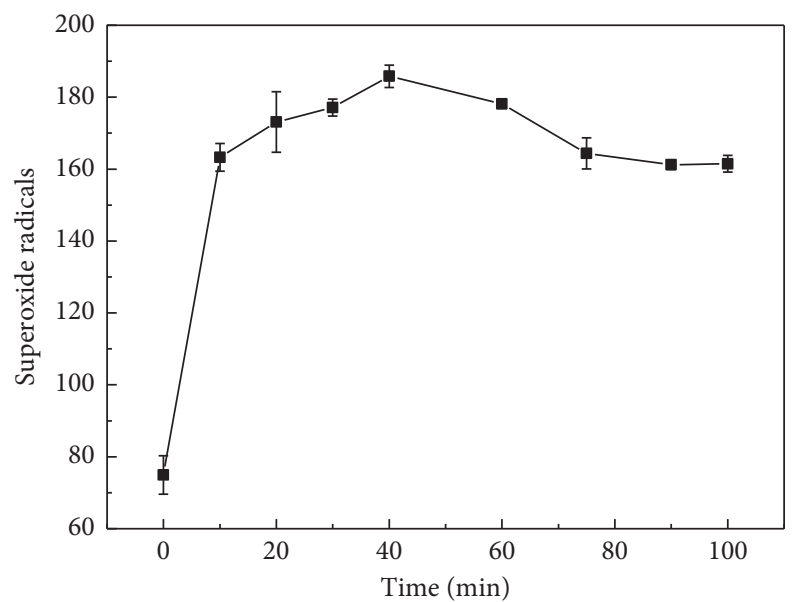

FIGURE 5: The effect of ultrasonic time on superoxide radicals.

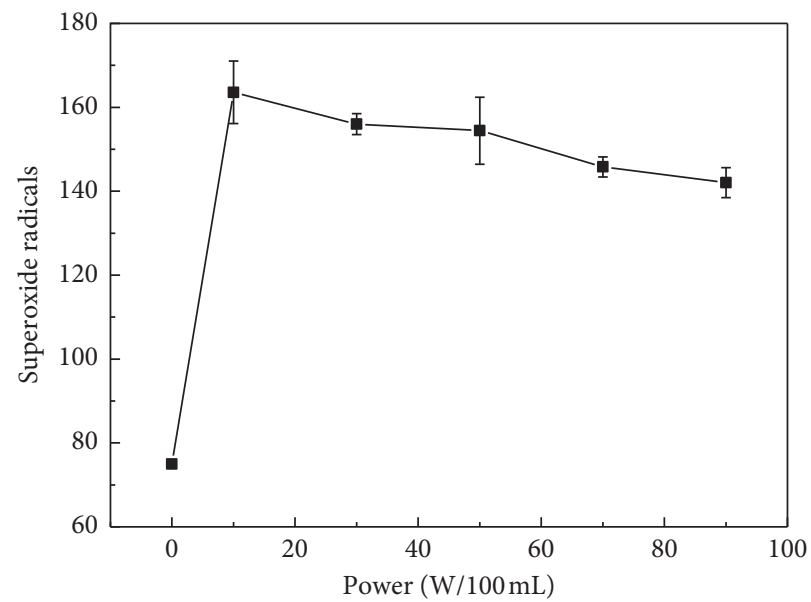

Figure 6: The effect of ultrasonic power on superoxide radicals.

TABLE 2: Changes in the free amino acids in the vinegar during the ageing process.

\begin{tabular}{|c|c|c|c|c|c|c|c|}
\hline \multirow{2}{*}{ Amino acids } & \multicolumn{6}{|c|}{ Amino acid content $(\mathrm{mg} / 100 \mathrm{~mL})$} & \multirow{2}{*}{ Taste } \\
\hline & Fresh & 12 months & 18 months & 32 months & 44 months & Ultrasonic treated & \\
\hline Asp & 82.43 & 67.24 & 51.90 & 48.03 & 46.76 & 52.22 & Sour \\
\hline Glu & 115.64 & 114.61 & 120.12 & 127.85 & 134.72 & 128.96 & Fresh \\
\hline $\mathrm{Val}^{\mathrm{E}}$ & 77.15 & 66.66 & 63.48 & 58.54 & 48.86 & 65.65 & Astringent \\
\hline $\mathrm{Leu}^{\mathrm{E}}$ & 107.87 & 92.73 & 85.18 & 82.42 & 78.73 & 89.50 & Astringent \\
\hline Gly & 91.65 & 70.70 & 66.49 & 63.85 & 61.84 & 64.73 & Strong sweet \\
\hline Ala & 118.64 & 103.56 & 94.81 & 90.06 & 83.65 & 94.46 & Strong sweet \\
\hline $\operatorname{Thr}^{\mathrm{E}}$ & 42.13 & 41.30 & 38.39 & 35.48 & 32.73 & 38.57 & Sweet \\
\hline Ser & 52.49 & 45.15 & 44.46 & 41.94 & 40.89 & 42.27 & Sweet \\
\hline $\mathrm{Met}^{\mathrm{E}}$ & 13.36 & 14.39 & 13.65 & 12.57 & 9.29 & 12.06 & Sweet \\
\hline $\mathrm{Phe}^{\mathrm{E}}$ & 34.18 & 31.83 & 45.29 & 39.79 & 45.45 & 39.56 & Sweet \\
\hline Pro & 61.46 & 38.97 & 43.16 & 32.81 & 35.68 & 36.89 & Sweet \\
\hline Lys $^{\mathrm{E}}$ & 45.65 & 36.55 & 37.30 & 27.95 & 21.19 & 27.21 & Slight sweet \\
\hline $\mathrm{Ile}^{\mathrm{E}}$ & 47.59 & 51.91 & 45.73 & 40.61 & 37.45 & 46.39 & Bitter \\
\hline Arg & 62.87 & 50.75 & 32.20 & 34.40 & 29.21 & 33.86 & Bitter \\
\hline Tyr & 49.81 & 35.48 & 30.72 & 31.44 & 27.78 & 31.29 & Slight bitter \\
\hline His & 27.77 & 15.00 & 16.18 & 11.57 & 10.12 & 16.44 & Slight bitter \\
\hline $\operatorname{Trp}^{\mathrm{E}}$ & 10.48 & 14.32 & 17.63 & 17.54 & 13.52 & 14.88 & Slight bitter \\
\hline Total & 1041.35 & 891.18 & 836.69 & 796.85 & 757.87 & 843.94 & \\
\hline
\end{tabular}

E represents the essential amino acids, $T$ represents the total amino acids, and $\mathrm{N}$ represents the nonessential amino acids. 


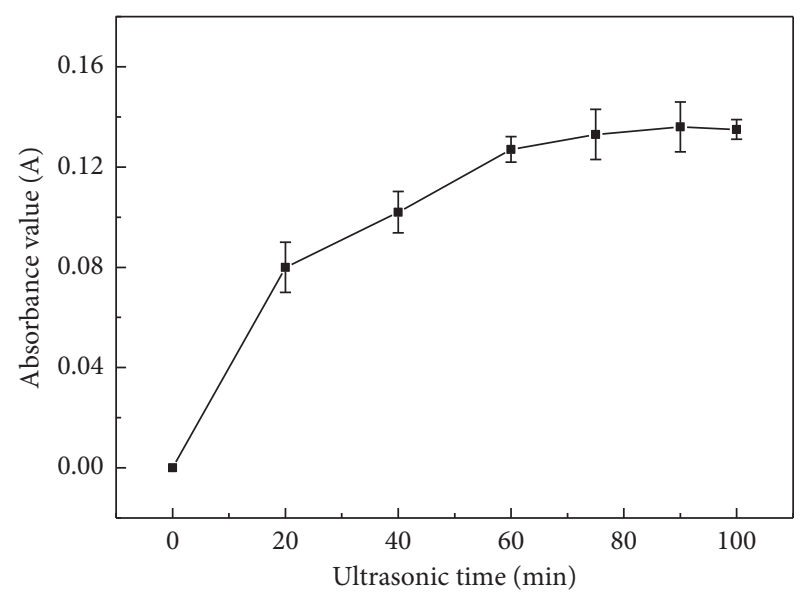

FIGURE 7: The effect of ultrasonic time on browning intensity.

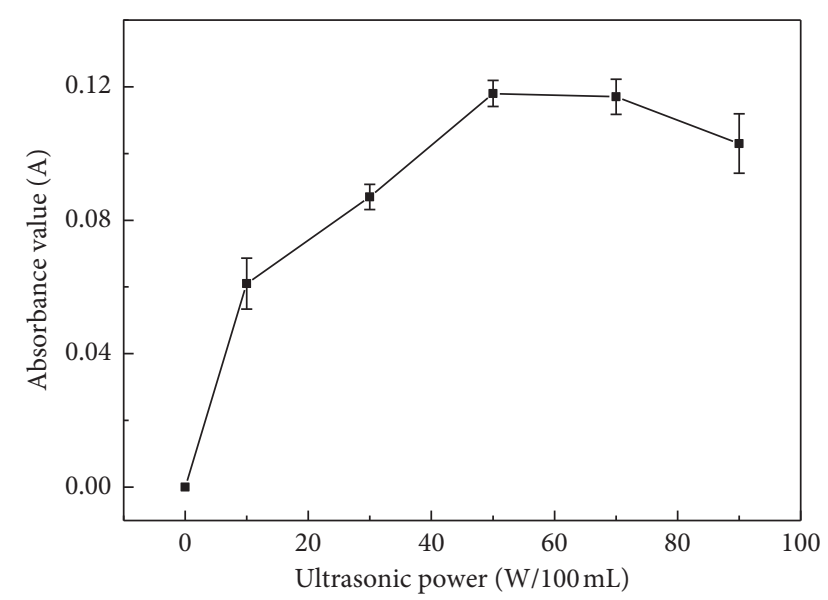

Figure 8: The effect of ultrasonic power on browning intensity.

the degree of browning increased slowly after $60 \mathrm{~min}$ of sonication. In Figure 8, with the ultrasonic power rise, the degree of browning of the simulated system showed a tendency to increase first and then decrease and reached the maximum when the ultrasonic power density was $50 \mathrm{~W} / 100 \mathrm{~mL}$. This result is consistent with our previous experimental conclusions [9]. The apparent increase in the absorbance indicates that the midterm product of the Maillard reaction is accumulating. This difference could be related to the acoustic cavitation. Since this cavitation activity can be viewed as a dramatic concentration of acoustic energy resulting in localized high stresses, temperatures, and/or fluid velocities, its biological consequences should be understood by those who are trying to either optimize or minimize its effects [47]. Additionally, the violent collapse of bubbles can generate exceedingly steep heat and pressure, which can produce free radicals and so forth [48], and then small molecules can rearrange [4]. The results show that the ultrasonic wave can strengthen the fructose-glycine Maillard reaction, promote the system browning, and induce the middle stage and the formation of advanced stage products.

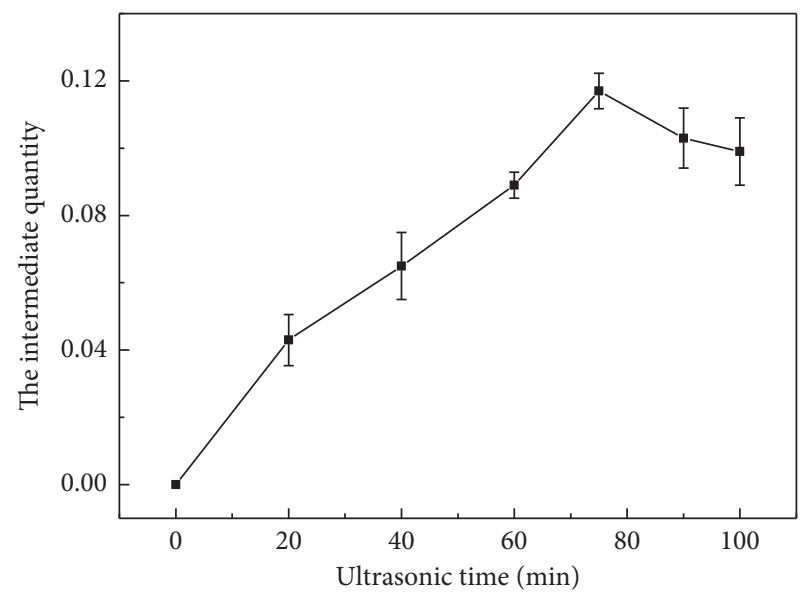

FIgURE 9: The effect of ultrasonic time on intermediate quantity.

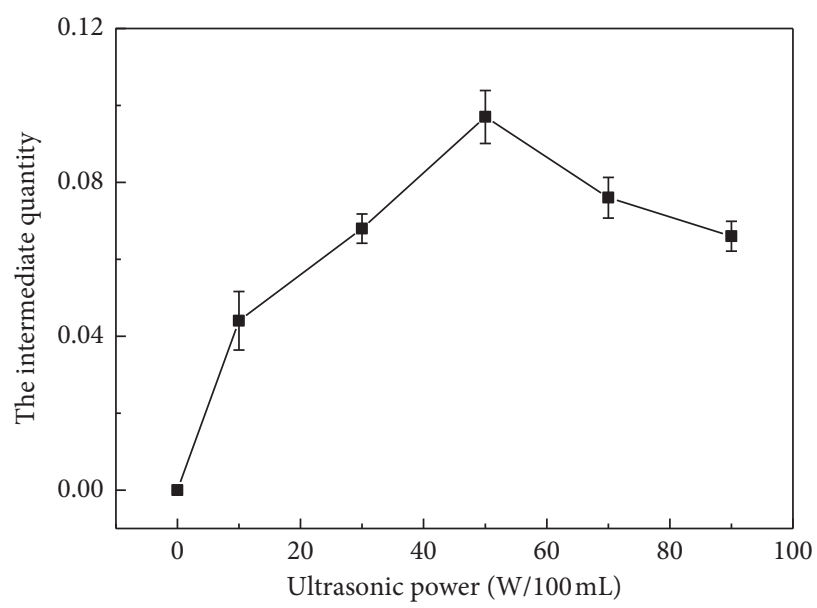

FIGURE 10: The effect of ultrasonic power on intermediate quantity.

3.5. Effect of Ultrasound on the Amount of the Intermediate Products in Maillard Reaction. In the Maillard reaction process, there are some noncolor intermediates, such as small molecules of ketones and aldehydes, which are also important indicators of the Maillard reaction [34]. As can be seen from Figures 9 and 10, without ultrasonic processing, the intermediate product of the Maillard reaction has not been detected in the simulation system solution. Applying the ultrasonic vinegar sample, however, the intermediate product of the reaction significantly altered. Results (Figures 9 and 10) showed that accompanied with the ultrasonic time and ultrasonic power rise, the median product of the Maillard reaction increased gradually, reaching the maximum at $75 \mathrm{~min}$ and $50 \mathrm{~W} / 100 \mathrm{~mL}$, respectively. The change of Maillard intermediates may largely be due to the fact that ultrasound can induce some oxygen ions and hydroxyl radicals in the aqueous solution [49], and the production and rearrangement of these oxygen ions and hydroxyl radicals can promote the Maillard reaction of the fructoseglycine simulation system, leading to the production of intermediates. The degree of browning and the number of intermediate products in the ultrasonic treatment 
simulation system can be used to prove the ultrasonic catalytic Maillard reaction.

\section{Conclusion}

In this study, under the conditions of ultrasonic processing, the changes of Zhenjiang vinegar physiochemical index, such as color, reducing sugar, and amino acid, are consistent with those of natural ageing. In addition, the sonicated vinegar produces hydroxyl radicals, which generally appear to increase with increasing ultrasound time and ultrasound power. The present study reveals that the mechanism of ultrasonic ageing lies in the cavitation of ultrasound to induce the generation of radicals, such as hydroxyl radicals and superoxide radicals, catalyze the oxidation reaction in vinegar, and verify it by the fructose-glycine Maillard simulation system. The results showed that ultrasonic treatment had positive impacts on the vinegar ageing process. For the future, much research is needed to determine the exact role of ultrasound in vinegar ageing.

\section{Data Availability}

All data generated or analyzed during this study are included in this published article.

\section{Conflicts of Interest}

All authors declare no conflicts of interest.

\section{Authors' Contributions}

Xiaoke Ma and Tingting Li contributed equally to this study.

\section{Acknowledgments}

This research was supported by a grant from Modern Agriculture-Key and General Projects (BE2018368) and the Key Technology R\&D Program of Zhenjiang (no. SH2019011).

\section{References}

[1] S. Baroni, R. Consonni, G. Ferrante, and S. Aime, "Relaxometric studies for food characterization: the case of balsamic and traditional balsamic vinegars," Journal of Agricultural and Food Chemistry, vol. 57, no. 8, pp. 3028-3032, 2009.

[2] L. Solieri and P. Giudici, Vinegars of the World, Springer, Berlin, Germany, 2009.

[3] Z. Zhou, S. Liu, X. Kong et al., "Elucidation of the aroma compositions of Zhenjiang aromatic vinegar using comprehensive two dimensional gas chromatography coupled to time-of-flight mass spectrometry and gas chromatographyolfactometry," Journal of Chromatography A, vol. 1487, pp. 218-226, 2017.

[4] T. Yang, F. Juan, and D. Sun, "Advances in wine ageing technologies for enhancing wine quality and accelerating wine ageing process," Critical Reviews in Food Science and Nutrition, vol. 6, pp. 817-835, 2014.

[5] A. C. Chang, "The effects of gamma irradiation on rice wine maturation," Food Chemistry, vol. 83, pp. 323-327, 2003.
[6] A. Chang, "Study of ultrasonic wave treatments for accelerating the aging process in a rice alcoholic beverage," Food Chemistry, vol. 92, no. 2, pp. 337-342, 2005.

[7] A. C. Chang and F. C. Chen, "The application of $20 \mathrm{kHz}$ ultrasonic waves to accelerate the aging of different wines," Food Chemistry, vol. 79, no. 4, pp. 501-506, 2002.

[8] N. Masuzawa, E. Ohdaira, and M. Ide, "Effects of ultrasonic irradiation on phenolic compounds in wine," Japanese Journal of Applied Physics, vol. 39, no. 5B, pp. 2978-2979, 2000.

[9] Z. Wang, T. Li, F. Liu et al., "Effects of ultrasonic treatment on the maturation of Zhenjiang vinegar," Ultrasonics Sonochemistry, vol. 39, pp. 272-280, 2017.

[10] X. A. Zeng, S. J. Yu, L. Zhang, and X. D. Chen, "The effects of AC electric field on wine maturation," Innovative Food Science \& Emerging Technologies, vol. 9, no. 4, pp. 463-468, 2008.

[11] A. C. Soria and M. Villamiel, "Effect of ultrasound on the technological properties and bioactivity of food: a review," Trends in Food Science \& Technology, vol. 21, no. 7, pp. 323-331, 2010.

[12] J. Li, J. Jiang, and Y. Fu, "Superhigh pressure process for wine ageing," CN 1241629 (A). European Patent Application, 2000.

[13] Y. Peng, R. Gan, H. Li et al., "Absorption, metabolism, and bioactivity of vitexin: recent advances in understanding the efficacy of an important," Critical Reviews in Food Science and Nutrition, vol. 27, pp. 1-16, 2020.

[14] F. Chemat, M. K. Zill-e-Huma, and M. K. Khan, “Applications of ultrasound in food technology: processing, preservation and extraction," Ultrasonics Sonochemistry, vol. 18, no. 4, pp. 813-835, 2011.

[15] L. Zheng and D.-W. Sun, "Innovative applications of power ultrasound during food freezing processes-a review," Trends in Food Science \& Technology, vol. 17, no. 1, pp. 16-23, 2006.

[16] V. Jiranek, P. Grbin, A. Yap, M. Barnes, and D. Bates, "High power ultrasonics as a novel tool offering new opportunities for managing wine microbiology," Biotechnology Letters, vol. 30 , no. 1, pp. 1-6, 2008.

[17] J. F. G. MartíN and D.-W. Sun, "Ultrasound and electric fields as novel techniques for assisting the wine ageing process: the state-of-the-art research," Trends in Food Science \& Technology, vol. 33, pp. 40-53, 2013.

[18] A. J. Saterlay and R. G. Compton, "Sonoelectroanalysis-an overview," Fresenius' Journal of Analytical Chemistry, vol. 367, no. 4, pp. 308-313, 2000.

[19] M. Ashokkumar, "The characterization of acoustic cavitation bubbles-an overview," Ultrasonics Sonochemistry, vol. 18, no. 4, pp. 864-872, 2011.

[20] E. Maisonhaute, C. Prado, P. C. White, and R. G. Compton, "Surface acoustic cavitation understood via nanosecond electrochemistry. Part III: shear stress in ultrasonic cleaning," Ultrasonics Sonochemistry, vol. 9, no. 6, pp. 297-303, 2002.

[21] L. Pripis-nicolau, G. De revel, A. Bertrand, and A. Maujean, "Formation of flavor components by the reaction of amino acid and carbonyl compounds in mild conditions," Journal of Agricultural and Food Chemistry, vol. 48, no. 9, pp. 3761-3766, 2000.

[22] M. Casale, M.-J. Sáiz Abajo, J.-M. González Sáiz, C. Pizarro, and M. Forina, "Study of the aging and oxidation processes of vinegar samples from different origins during storage by nearinfrared spectroscopy," Analytica Chimica Acta, vol. 557, no. 1-2, pp. 360-366, 2006.

[23] H. Brückner, J. Justus, and J. Kirschbaum, "Saccharide induced racemization of amino acids in the course of the 
Maillard reaction,” Amino Acids, vol. 21, no. 4, pp. 429-433, 2001.

[24] S. C. Shen and S. B. Wu, "Maillard browning in ethanolic solution," Journal of Food Science, vol. 69, no. 4, Article ID FCT273-FCT279, 2004.

[25] S. C. Shen, K. C. Tseng, and S. B. Wu, "An analysis of Maillard reaction products in ethanolic glucose-glycine solution," Food Chemistry, vol. 102, no. 1, pp. 281-287, 2007.

[26] M. J. Gómez-m Guez, M. Gmez-m guez, I. M. Vicario, and F. J. Heredia, "Assessment of colour and aroma in white wines vinifications: effects of grape maturity and soil type," Journal of Food Engineering, vol. 79, pp. 758-764, 2007.

[27] Z. Chunyan, "Improvement of flavor and color of vinegar produced by submerged fermentation," China Brewing, vol. 19, pp. 79-81, 2008.

[28] L. I. Gang, "Influence of several anti-oxidizing agent upon the persimmon vinegar color," Journal of Anhui Agricultural Sciences, vol. 34, pp. 651-654, 2006.

[29] B. Gordillo, M. J. Cejudo-Bastante, F. J. Rodríguez-Pulido et al., "Impact of adding white pomace to red grapes on the phenolic composition and color stability of syrah wines from a warm climate," Journal of Agricultural and Food Chemistry, vol. 62, no. 12, pp. 2663-2671, 2014.

[30] O. S. Pérez-magari and M. L. Gonz lez-san JOS, "Polyphenols and colour variability of red wines made from grapes harvested at different ripeness grade," Food Chemistry, vol. 96, pp. 197-208, 2006.

[31] Z. Xiaodong, Y. Huizhong, and L. Zhiyi, "Relationship between strength of hydrodynamic cavitation and amount of induced hydroxyl radical," Journal of Chemical Industry and Engineering:China, vol. 33, pp. 799-809, 2007.

[32] H.-J. Zhang and H.-S. Xia, "Measurement of hydroxyl radical induced by cavitation of HIFU," Guangzhou Chemical Industry, vol. 36, pp. 47-48, 2008.

[33] F. Gu, J. M. Kim, K. Hayat, S. Xia, B. Feng, and X. Zhang, "Characteristics and antioxidant activity of ultrafiltrated Maillard reaction products from a casein-glucose model system," Food Chemistry, vol. 117, no. 1, pp. 48-54, 2009.

[34] E. H. Ajandouz, L. S. Tchiakpe, F. D. Ore, A. Benajiba, and A. Puigserver, "Effects of $\mathrm{pH}$ on caramelization and maillard reaction kinetics in fructose-lysine model systems," Journal of Food Science, vol. 66, no. 7, pp. 926-931, 2001.

[35] H. M. Chawla and S. N. Sahu, "Effect of spice essential oils on Maillard browning model reaction of glucose and glycine: an UV-visible and reverse phase HPLC analysis," Journal of Food Science and Technology-Mysore-, vol. 44, pp. 602-606, 2007.

[36] J. Oszmiański, A. Wojdylo, and J. Kolniak, "Effect of enzymatic mash treatment and storage on phenolic composition, antioxidant activity, and turbidity of cloudy apple juice," Journal of Agricultural \& Food Chemistry, vol. 57, pp. 70787085, 2009.

[37] J. Oszmiański, A. Wojdyło, and J. Kolniak, "Effect of pectinase treatment on extraction of antioxidant phenols from pomace, for the production of puree-enriched cloudy apple juices," Food Chemistry, vol. 127, no. 2, pp. 623-631, 2011.

[38] X. Z. Lin, W. X. Li, Z. C. Liang, X. Y. Ren, and Z. G. He, "Study on the loquat fruit vinegar of accelerating maturity technology by ultrasonic," China Condiment, vol. 10, pp. 23-26, 2009.

[39] Z. Wang, S. Shao, S. Zhao, and M. A. Haile, "Application of principal component analysis in comprehensive assessment of ultrasound treatment on vinegar," Journal of Food Science \& Biotechnology, vol. 34, pp. 73-79, 2015.

[40] Q.-A. Zhang, H. Shen, X.-H. Fan, Y. Shen, X. Wang, and Y. Song, "Changes of gallic acid mediated by ultrasound in a model extraction solution," Ultrasonics Sonochemistry, vol. 22, pp. 149-154, 2015.

[41] D. Tpa, J. C. Tilak, K. K. Boloor, K. S. Sane, S. S. Ghaskadbi, and R. D. Lele, "Free radicals and antioxidants in human health," The Journal of the Association of Physicians in Indians, vol. 52, pp. 794-804, 2004.

[42] B. Halliwell, "Role of free radicals in the neurodegenerative diseases," Drugs \& Aging, vol. 18, no. 9, p. 685, 2001.

[43] S. Jie, The Study for Influence of Quality of Rice Wine by Ultrasonic Treatment and its Mechanism, Zhejiang University, Hangzhou, China, 2013.

[44] H. Zhang and F. Z. Ren, "Advances in determination of hydroxyl and superoxide radicals," Spectroscopy \& Spectral Analysis, vol. 29, p. 1093, 2009.

[45] J.-S. Kim, "Study on maillard reaction products derived from aqueous and ethanolic fructose-Glycine and its oligomer solutions," Preventive Nutrition and Food Science, vol. 15, no. 4, pp. 297-303, 2010.

[46] J.-S. Kim and Y.-S. Lee, "Characteristics and antioxidant activity of Maillard reaction products from fructose-glycine oligomer," Food Science and Biotechnology, vol. 19, no. 4, pp. 929-940, 2010.

[47] H. Liu and C. Hsieh, "Single-transducer dual-frequency ultrasound generation to enhance acoustic cavitation," Ultrasonics Sonochemistry, vol. 16, no. 3, p. 431, 2009.

[48] A. Strybulevych, V. Leroy, M. G. Scanlon, and J. H. Page, "Characterizing a model food gel containing bubbles and solid inclusions using ultrasound," Soft Matter, vol. 3, no. 11, pp. 1388-1394, 2007.

[49] M. Corzo-Martínez, A. Montilla, R. Megías-Pérez, A. Olano, F. J. Moreno, and M. Villamiel, "Impact of high-intensity ultrasound on the formation of lactulose and Maillard reaction glycoconjugates," Food Chemistry, vol. 157, pp. 186192, 2014 